\title{
Eddy-Current Modeling of a Continous Conductivity Profile Resulting From a Diffusion Process
}

\author{
M. Lambert, F. Nouguier and R. Zorgati
}

Département de Recherche en Electromagnétisme, Laboratoire des Signaux et Systèmes UMR8506 (CNRS-Supélec-Univ Paris-Sud), 91192 Gif-sur-Yvette cedex, France

\begin{abstract}
:
This paper deals with the modelization of eddy-current measurements over combustion turbine blade coatings affected by depletion of aluminum. First, we model the response of an eddy-current coil over a layered metallic structure with a top over-aluminized coating by extending the analytical Uzal-Rose's model for one hyperbolic tangent conductivity profile to a conductivity profile using two hyperbolic tangents for taking inward and outward depletion of aluminum inside the coating into account. Results obtained with this model are similar to those obtained with a numerical multilayer model but with a reduced computing time.
\end{abstract}

Keywords : coils, eddy current testing, electrical conductivity measurement, inverse problems, probes

\section{Introduction}

The modelization of the response of a sensor in the presence of conductive material of different geometry is of great interest as exemplified in [1] where the interaction of an Eddycurrent coil with a right-angled conductive wedge is dealt with, in [2] in which the impedance calculation of a bobbin coil in a conductive tube with eccentric walls is treated. Some others works proposed some ideas to work with arbitrary shaped coil as in [3] whereas in [4] the case of the inductance of a coil in a nonhomogenous surrounding is dealt with. Here the eddy-current modeling of the interaction of a

coil with a continous conductivity profile resulting from a diffusion process is proposed.

In the electrical industry, power production using gas turbines has to be developed in a significant way. In order to achieve a good economical performance, the thermal efficiency of gas turbines has been improved by increasing the firing temperatures. Large increase by hundreds of degrees of gas temperature has been made possible using high technologies regarding both cooling of the hot section components and protection against hot corrosion and high temperature oxidation of the surface of some components, like blades in highpressure section.

This protection involves specific coatings using Nibased super-alloys where aluminum and chrome, contained in precise proportion, form protective $\mathrm{Al} 2 \mathrm{O} 3$ or $\mathrm{Cr} 2 \mathrm{O} 3$ surface oxides against corrosive attacks. Yet, advanced blades protected by a top over-aluminized coating are affected by a significant inward and outward depletion of aluminum. Inward depletion is due to migration of aluminum from the coating to the blade substrate, 
induced by differences of conductivities between the two layers, in order to restore the thermodynamical equilibrium. Outward depletion is due to migration of aluminum in the coating towards the surface to reform the protective oxides layer which spalls off during hostile conditions operation. Unfortunately, when losing aluminum, advanced top over-aluminized coating degrades and, if not timely repaired, this might lead to blade failure. Benefits of a higher operating temperature is then counteracted by possible limited operating life and high replacement cost. It is hence necessary to predict remaining blade coating life by assessing condition and quality of the coating. Expected reduction of shut-downs caused by blade coating failure then should contribute to optimize the maintenance of combustion turbines.

By providing information related to the electrical conductivity depth profile, the multi-frequency Eddy Current (EC) non-destructive technique can be useful for reaching that goal. The paper is organized as follows. The configuration at hand is described in section II. Section III is devoted to the solution of the direct problem which aims at modeling the response of an EC coil above a layered metal structure protected by a top over-aluminized coating degraded by inward and outward diffusion of aluminum. To take such diffusion process of aluminum inside the blade into account, we develop a model where the conductivity profile, which varies continuously with depth in the blade, is described via two hyperbolic tangents. We hence extend the UzalRose's model [5]-[7] based on one hyperbolic tangent conductivity profile. Comparison of results obtained with this model and with those obtained with a numerical multi-layer model, based on discretization of the conductivity profile into a number of layers of constant conductivity are presented in section VI.

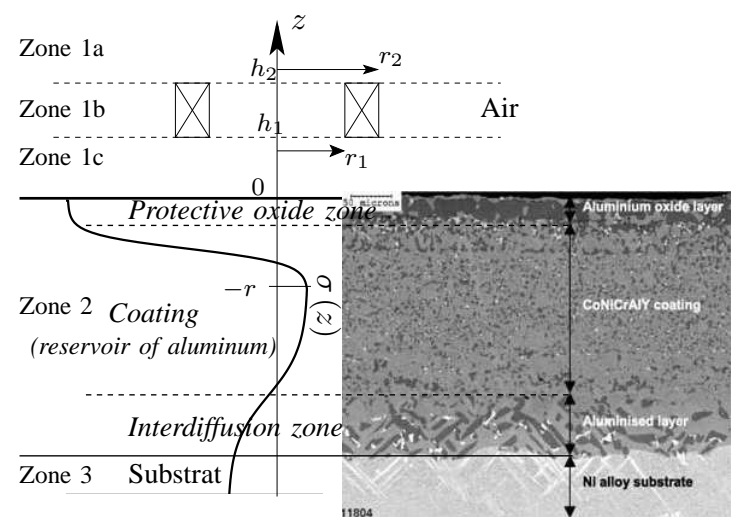

Fig. 1. Typical configuration of measurement with a theoretical conductivity profile with inward and outward diffusion of aluminum (left); example of a real diffusion process (right).

\section{Eddy Current Model for Turbine Blade CoAting}

Save its convex and concave parts, a blade turbine made of a substrate and a coating is considered as a doubly layered isotropic, linear, homogeneous, nonmagnetic and conductive half-space. The top overaluminized coating protects the substrate by forming a thin adherent oxide and degrades in-service by depletion of aluminum as commented upon before. The conductivity of the coating layer is a continuous function of depth $z$ while the conductivity of the substrate layer is a given constant (cf. Fig. 1).

Above the blade, an EC probe with a circular coil of rectangular cross-section with axis perpendicular to the blade surface is operated at frequencies in the range of a few hundreds of $\mathrm{kHz}$ to a few tens of $\mathrm{MHz}$ using a scanning frequency procedure. In this section, we aim at solving the related direct problem consisting of modeling the EC impedance coil response over a layered metallic blade structure and taking the double diffusion of aluminum into account when the conductivity depth profile is given.

Following the approach in [5]-[8], based on the 
Cheng-Dodd-Deeds model [9], [10], the two main steps for establishing the model are determining the vector potential and then calculating the coil impedance from $Z=V / I$ after computing the electrical field by $\mathbf{E}(\mathbf{r})=$ $-j \omega \mathbf{A}(\mathbf{r})$. From the fundamental result which gives the impedance of a coil located above a two-layer metal whose upper layer conductivity varies continuously with depth, one can calculate the variation of impedance for a large number of configurations.

\section{TheORETICAL FORMULATION OF THE PROBLEM}

\section{A. General formulation for a continuous profile}

The first step consists of calculating the magnetic vector potential in air expressed as $\mathbf{A}(\mathbf{r})=\mathbf{A}_{\text {Source }}(\mathbf{r})+$ $\mathbf{A}_{\text {ec }}(\mathbf{r})$, where $\mathbf{A}_{\text {Source }}(\mathbf{r})$ is the primary potential due to the time-harmonic source current of the coil $I \exp (\mathrm{j} \omega t)$ in an unbounded space and $\mathbf{A}_{\mathrm{ec}}(\mathbf{r})$ is the secondary potential originated from the EC induced within the conductive material. Assuming axial symmetry and choosing the cylindrical coordinates centered on the axis of the coil leads to a scalar problem, all vector quantities being expressed with the only non-null component, the azimuthal one, as $\mathbf{A}(\mathbf{r})=A(r, z) \mathbf{u}_{\theta}$. The expression of the primary field $A_{\text {Source }}(r, z)$ due to a $N_{\text {turn }}$ coil can be found in [10] and the secondary field $A_{\text {ec }}(r, z)$ is solution of the diffusive equation (see [11] for a justification of eddy currents model for the Maxwell equations):

$$
\Delta A_{\mathrm{ec}}(r, z)=\mathrm{j} \omega \mu_{0} \sigma(z) A_{\mathrm{ec}}(r, z)
$$

with

$$
\Delta=\frac{\partial^{2}}{\partial r^{2}}+\frac{1}{r} \frac{\partial}{\partial r}+\frac{\partial^{2}}{\partial z^{2}}-\frac{1}{r^{2}},
$$

where the continuously varying conductivity profile is given by $\sigma(z), \mu_{0}$ being the permeability of the air and $\omega=2 \pi f$ the angular frequency and $f$ the frequency (in
$\mathrm{Hz})$. Let us defined the skin depth $\delta(z)$ as

$$
\delta(z)=\sqrt{\frac{2}{\omega \mu_{0} \sigma(z)}} .
$$

Equation (1) can be solved by separation of variables letting $A_{\mathrm{ec}}(r, z)=R(r) W(z)$. The two ordinary differential equations for the $r$ and the $z$ dependence, respectively, follows as:

$$
\begin{gathered}
\frac{\partial^{2}}{\partial r^{2}} R(r)+\frac{1}{r} \frac{\partial}{\partial r} R(r)+\left(a^{2}-\frac{1}{r^{2}}\right) R(r)=0 \\
\frac{\partial^{2}}{\partial z^{2}} W(z)=\left[a^{2}+\mathrm{j} \omega \mu_{0} \sigma(z)\right] W(z) .
\end{gathered}
$$

Whilst the solution of (4) is classically obtained using first- and second-kind Bessel functions of the first order, the solution of (5) constitutes a more challenging task and can be performed by an analytical method for some particular conductivity profiles. Following the path presented in [8] the general solution of (5) is given by

$$
W(z)=C F_{1}(f(z))+B F_{2}(f(z))
$$

where $F_{1}$ and $F_{2}$ are functions related to the choice of the profile $\sigma(z), f(z)$ originating from possible transformations of the independent variable that take place during the solution of (5). In so doing the expression of the azimuthal component of the potential vector is given in a general form as

$$
\begin{aligned}
A_{1 c}(r, z)= & \int_{0}^{+\infty} \frac{\mu N_{\mathrm{turn}} I \mathcal{I}\left(a r_{1}, a r_{2}\right) e^{a z} J_{1}(a r)}{2 a^{3}\left(h_{2}-h_{1}\right)\left(r_{2}-r_{1}\right)} \\
& \times\left(e^{-a h_{1}}-e^{-a h_{2}}\right) d a+\int_{0}^{+\infty} C_{1} e^{-a z} J_{1}(a r) \mathrm{d} a \\
& \forall z \in[0 ;+\infty[
\end{aligned}
$$

$$
A_{2}(r, z)=\int_{0}^{+\infty}\left[C_{2} F_{1}(f(z))+B_{2} F_{2}(f(z))\right] J_{1}(a r) \mathrm{d} a
$$

$$
\forall z \in[-r ; 0]
$$

$\left.\left.A_{3}(r, z)=\int_{0}^{+\infty} B_{3} F_{3}(g(z)) J_{1}(a r) d a \quad \forall z \in\right]-\infty ;-r\right]$ 
where $f(z)$ and $g(z)$ are two different functions of $z$ and where $\mathcal{I}\left(x_{1}, x_{2}\right)$ is given by

$$
\mathcal{I}\left(a r_{1}, a r_{2}\right)=\int_{x_{1}}^{x_{2}} x J_{1}(x) \mathrm{d} x .
$$

Once $A_{1 c}(r, z)$ is known, $Z$ is deduced as

$$
\begin{aligned}
Z=K \int_{0}^{+\infty} \frac{\mathcal{I}\left(a r_{1}, a r_{2}\right)^{2}}{a^{6}}\left[2 \left(e^{-a\left(h_{2}-h_{1}\right)}-1+a\left(h_{2}-h_{1}\right)\right.\right. \\
\left.+\left(e^{-a h_{2}}-e^{-a h_{1}}\right)^{2} \phi(a)\right] d a
\end{aligned}
$$

with

$$
\begin{gathered}
\phi(a)=\frac{C_{1}}{\mathcal{K}}, \\
\mathcal{K}=\frac{\mu N_{\text {turn }} I \mathcal{I}\left(a r_{1}, a r_{2}\right)\left(e^{-a h_{1}}-e^{-a h_{2}}\right)}{2 a^{3}\left(h_{2}-h_{1}\right)\left(r_{2}-r_{1}\right)} \\
K=\frac{j \omega \pi \mu N_{\text {turn }}^{2}}{\left(h_{2}-h_{1}\right)^{2}\left(r_{2}-r_{1}\right)^{2}} .
\end{gathered}
$$

The expressions of $C_{1}, C_{2}, B_{2}$ and $B_{3}$ are obtained from the continuity conditions of the quantities and/or their derivatives with respect to depth and/or their cancellation at $\pm \infty$. Denoting all such quantities as

$$
\begin{aligned}
& L=F_{1}(f(z=0)) ; \\
& M=F_{2}(f(z=0)) ; \\
& N=\left.F_{1}^{\prime}(f(z))\right|_{z=0} ; \\
& O=\left.F_{2}^{\prime}(f(z))\right|_{z=0} \\
& P=F_{1}(f(z=-r)) ; \\
& Q=F_{2}(f(z=-r)) \\
& R=\left.F_{1}^{\prime}(f(z))\right|_{z=-r} ; \\
& S=\left.F_{2}^{\prime}(f(z))\right|_{z=-r} \\
& T=F_{3}(g(z=-r)) ; \\
& U=\left.F_{3}^{\prime}(g(z))\right|_{z=-r}
\end{aligned}
$$

where' means derivative with respect to $z$ and solved the corresponding linear system, the expression of $\phi(a)$ is obtained as

$$
\phi(a)=\frac{(a M-O)(R T-P U)+(a L-N)(Q U-S T)}{(a M+O)(R T-P U)+(a L+N)(Q U-S T)}
$$

Applying such an approach, analytical solutions of (5) for a continuous conductivity function $\sigma(z)$ have been proposed when $\sigma(z)$ varies linearly, quadratically or exponentially [8] and when $\sigma(z)$ is varying as an hyperbolic tangent [5]. In [12] the authors are interested in the case of double-layer media with depth-varying magnetic properties where the permeability is continuously varying as a function of the exponential of the depth whereas the case of a layered medium where both the conductivity and the permeability are continuously varying as a power of the depth is adressed in [13].

\section{B. Series expansion of the formulation (11)}

According to the method described in [14] the integral expression of the impedance (11) can be replaced by a series expansion (26) which allows a better control of the convergence and a faster evaluation at least at low frequency. This method is based on a reduction of the solution region in the radial direction at a distance $b$ where a homogeneous Dirichlet condition is imposed onto the radial component of magnetic field. The integrand of (11) is slightly modified and only evaluated at discrete values $a_{i}$ of $a$, the latter being obtained from $J_{1}\left(a_{i} b\right)=0$. The number $M$ of $a_{i}$ to be taken into account depends on the sought accuracy on the value of $Z$.

$$
\begin{aligned}
Z_{M} \approx 2 K \sum_{i=1}^{M} & \frac{\mathcal{I}\left(a_{i} r_{1}, a_{i} r_{2}\right)^{2}}{\left[a_{i} b J_{0}\left(a_{i} b\right)\right]^{2} a_{i}^{5}} \\
& \times\left[\left(e^{-a_{i} h_{2}}-e^{-a_{i} h_{1}}\right)^{2} \phi\left(a_{i}\right)\right. \\
& \left.+2\left(e^{-a_{i}\left(h_{2}-h_{1}\right)}-1+a_{i}\left(h_{2}-h_{1}\right)\right)\right]
\end{aligned}
$$


(note that $Z_{M} \rightarrow Z$ when $M \rightarrow+\infty$ for sufficiently large $b)$.

\section{Particular formulation for a two-tanh-continous pro-} file

For the applications which we are interested in, the inward and outward diffusion are to be taken into account, so we suggest to apply the analytical method for dealing with (5) with a conductivity depth profile defined by two hyperbolic tangents, one for the inward diffusion and the other for the outward one, as follows:

$\sigma(z)=\left\{\begin{array}{l}\sigma_{12}+\Delta \sigma\left[1+\tanh \left(\frac{z+c_{1}}{2 v_{1}}\right)\right] \forall z \in[-r, 0] \\ \sigma_{2}-\Delta \sigma\left[1+\tanh \left(\frac{z+c_{2}}{2 v_{2}}\right)\right] \forall z<-r\end{array}\right.$

with $\Delta \sigma=\left(\sigma_{1}-\sigma_{12}\right) / 2$ and where $\sigma_{1}$ is the conductivity limit value related to the first profile when $z$ tends to $+\infty, \sigma_{2}$ is the conductivity limit value related to the second profile when $z$ tends to $-\infty$ and $\sigma_{12}$ is the joining conductivity limit value related to the first profile when $z$ tends to respectively $-\infty$ and to the second profile when $z$ tends to $+\infty$.

The joining point between the two sub-profiles, denoted $r$, is obtained by equating expressions of $\sigma(z)$ in (27) for $z=-r . v_{1}$ and $v_{2}$ can be seen as "the transition speed" of the conductivity in the $[-r, 0]$ and $[-\infty,-r]$ and $c_{1}$ and $c_{2}$ the depth corresponding to the inflection point. A typical profile of such a kind is shown in Fig. 1. In fact, such a modeling allows us to define a large class of various profiles with a small number of parameters adequately chosen. Let us notice that with such a formulation a continuous increasing (or decreasing) profile cannot be easily obtained even if, at the boundary of the two layers, the conductivity, function of depth, is allowed to be discontinuous.

For the case of a single tanh-profile all details can be found in [5] and in the following only the two tanhprofiles will be dealt with. In such a case it can be shown that the expression of the special functions $F_{1}, F_{2}$ and $F_{3}$ are

$$
\begin{gathered}
\begin{aligned}
& F_{1}\left(y_{2}(z)\right)= y_{2}^{\mu}(z)\left[1-y_{2}(z)\right]^{\nu} \\
& \times{ }_{2} F_{1}\left(\mu+\nu, \mu+\nu+1 ; 2 \mu+1 ; y_{2}(z)\right) \\
& F_{2}\left(y_{2}(z)\right)= y_{2}{ }^{\mu}(z)\left[1-y_{2}(z)\right]^{\nu} \\
& \times{ }_{2} F_{1}\left(\nu-\mu+1, \nu-\mu ; 1-2 \mu ; y_{2}(z)\right) \\
& F_{3}\left(y_{3}(z)\right)= y_{3}^{\lambda}(z)\left[1-y_{3}(z)\right]^{\tau} \\
& \times{ }_{2} F_{1}\left(\lambda+\tau, \lambda+\tau+1 ; 2 \lambda+1 ; y_{3}(z)\right) \\
& \text { with } \quad \\
& y_{2}(z)=\left(1+e^{-\frac{z+c_{1}}{v_{1}}}\right)^{-1} \\
& y_{3}(z)=\left(1+e^{-\frac{z+c_{2}}{v_{2}}}\right)^{-1} \\
& \mu=v_{1} \sqrt{a^{2}+\mathrm{j} \omega \mu_{0} \sigma_{12}} \\
& \nu=v_{1} \sqrt{a^{2}+\mathrm{j} \omega \mu_{0} \sigma_{1}} \\
& \lambda=v_{2} \sqrt{a^{2}+\mathrm{j} \omega \mu_{0} \sigma_{2}} \\
& \tau=v_{2} \sqrt{a^{2}+\mathrm{j} \omega \mu_{0} \sigma_{12}}
\end{aligned}
\end{gathered}
$$

${ }_{2} F_{1}(\alpha, \beta ; \gamma ; x)$ is the Gauss hypergeometric function defined as [15]

$$
{ }_{2} F_{1}(\alpha, \beta ; \gamma ; x)=\sum_{k=0}^{+\infty} \frac{(\alpha)_{k}(\beta)_{k}}{(\gamma)_{k}} \frac{x^{k}}{k !}
$$

where $(\alpha)_{k}$ is the Pochhammer symbol given by

$$
(\alpha)_{k}=\alpha(\alpha+1)(\alpha+2) \cdots(\alpha+k-1)
$$

Let us define the derivative of ${ }_{2} F_{1}(\alpha, \beta ; \gamma ; x)$ with respect to $x$ as [15]

$$
\frac{\partial}{\partial x}{ }_{2} F_{1}(\alpha, \beta ; \gamma ; x)=\frac{\alpha \beta}{\gamma}{ }_{2} F_{1}(\alpha+1, \beta+1 ; \gamma+1 ; x)
$$

Introducing the expressions of (28) in (24) and using the derivative's rules (32) allows to express the analytical formulation of the functions $L, M, N, O, P, Q, R, S, T, U$ (see $\S$ A for their analytical 
expressions) and then to obtain the expression of $\phi(a)$ through (25).

\section{BRIEF DESCRIPTION OF THE MULTI-LAYER}

\section{MODEL}

Proper results for numerically validating our approach in the two-tanh profile configuration do not appear in the literature. A classical scheme consisting in the approximation of the continuously varying conductivity profile by slicing the coating layer into a sufficiently high number of planar homogeneous layers of given thickness and constant conductivity is considered. It can be sketched as follows

1) the lower half-space ( $z<0$ in Fig. 1) is divided in two parts:

- the upper one (zone 2 in Fig. 1) is a nonhomogeneous slab of thickness $d$. It is divided into $N$ homogeneous sub-layers of constant thickness $\Delta(=d /(N-1))$ and conductivity $\sigma_{i}, i=1 \ldots, N-1$, such as to provide a good discretized description of $\sigma(z)$;

- the lower one (zone 3 in Fig. 1) is taken as a half-space of constant conductivity $\sigma(z=d)$;

2) the Fresnel reflection coefficient for a TE-polarized electromagnetic wave of the multi-layer structure is computed using the algorithm proposed in [10]. It can be shown (no detail is provided here) that such a coefficient is nothing but $\phi(a)$ in (11) or in $(26)$;

3) for each frequency $f$, a given $N$ and a given $d$ are chosen and the corresponding impedance $Z$ is computed using either (11) or (26);

This method should ensure, in theory, high accuracy of the solution. In practice, the high number of discretization layers requested leads to a method which might be unsuitable, despite its generality, due to increase of computing time with respect to the number of layers considered.

For our purpose and for sake of simplicity, let us consider a configuration for which we would like to compute the impedance $Z$ for a range of frequencies from $f_{\min }$ to $f_{\max }$. Then $d$ is chosen large enough to entirely include the varying part of $\sigma(z)$ and is kept constant for all frequencies. Here $d$ has been arbitrarily chosen as:

$$
d=\sqrt{\frac{2}{\omega_{\min } \mu_{0} \sigma_{\min }}}
$$

with $\sigma_{\min }=\min \left(\sigma_{1}, \sigma_{12}, \sigma_{2}\right)$ and $\omega_{\min }=2 \pi f_{\min }, f_{\min }$ being the smallest frequency which we are interested in. Once $d$ has been fixed then $N$ is chosen large enough by trial-error scheme so that the corresponding impedance $Z_{N}$-obtained for $N$ layers- tends to the true impedance $Z$ at the higher frequency $f_{\max }$.

Such a choice is clearly not optimal and could be efficiently improved.

1) The thickness $\Delta_{i}$ of the $i^{\text {th }}$ sub-layer should be adapted to the local curvature of the profile $\sigma(z)$ -small when $\sigma(z)$ is rapidly varying, large when $\sigma(z)$ is almost constant.

2) Only the $M$ first sub-layers, those which are able to correctly describe the attenuation phenomena, have to be taken into account. As a matter of fact, knowing that eddy currents only penetrate at few skin depths in a metal plate and that the skin depth varies with respect to frequency (see (3)) the higher the frequency the smaller the number of sub-layers to take into account.

However for shake of simplicity such an adaptive scheme has not been applied in the following.

\section{NUMERICAL TREATMENT}

Numerically speaking the computation of (11) is difficult. As a matter of fact, even if we know that such 
an integral is convergent, $-\mathcal{I}\left(r_{1}, r_{2}\right)$ is decreasing and $\phi(a)$ plays the role of a reflection coefficient and then is bounded by 1 when $a$ goes to $+\infty$ - the theoretical formulation of $\phi(a)$ (25) involves the hypergeometric function (30) or its derivative (32) in its numerator and denominator.

The fast computation of the Gauss hypergeometric function ${ }_{2} F_{1}$ with complex parameters is a difficult task and is still the subject of significant research as exemplified in [16] and [17] with two different approaches. Both of them provide Fortran subroutines which have been used in our numerical simulations, [16] proposed a very general approach to compute all hypergeometric functions ${ }_{p} F_{q}$ (subroutine PFQ) whereas [17] focuses on the computation of the Gauss hypergeometric function ${ }_{2} F_{1}$ (subroutine HYP_2F1) we are interested in.

The numerical integration of (11) is performed using the DQAGI subroutine provided by IMSL.

\section{NUMERICAL VALIDATION}

\section{A. A single tanh profile [5]}

The validation of our approach is made by comparison with the results given in [5] for a single tanh profile. With reference to Fig. 1 the parameters of the measurement configuration are taken as: $r_{1}=1.3 \mathrm{~mm}$, $r_{2}=3.3 \mathrm{~mm}, h_{1}=0.5 \mathrm{~mm}, h_{2}=7.8 \mathrm{~mm}$ and $N_{\text {turn }}=580$. According to (27) the conductivity profile has the following parameters: $\sigma_{1}=1.509 \times 10^{7} \mathrm{~S} \mathrm{~m}^{-1}$, $\sigma_{12}=3.766 \times 10^{7} \mathrm{Sm}^{-1}, c_{1}=0.3 \mathrm{~mm}$ and $v_{1}=$ $0.1857 \mathrm{~mm}$.

A comparison of the results at three frequencies is shown in Table I for different values of $b$. A brief study of the convergence of the results with respect of the choice of the hyper-parameter $b$ is presented. A very good agreement is observed with [5]. (a) Real part of $\Delta Z$

\begin{tabular}{cccr}
\hline & $1 \mathrm{kHz}$ & $10 \mathrm{kHz}$ & $100 \mathrm{kHz}$ \\
\hline$[5]$ & 0.00817 & 0.02583 & -0.68836 \\
Numerical Integration & 0.008165 & 0.025809 & -0.68831 \\
$N=10$ & 0.008169 & 0.02585 & -0.68799 \\
$N=20$ & 0.008165 & 0.025823 & -0.68820 \\
\hline
\end{tabular}

(b) Imaginary part of $\Delta Z$

\begin{tabular}{crrr}
\hline & $1 \mathrm{kHz}$ & $10 \mathrm{kHz}$ & $100 \mathrm{kHz}$ \\
\hline$[5]$ & -0.00828 & -0.22571 & -1.49719 \\
Numerical Integration & -0.00828 & -0.22565 & -1.49637 \\
$N=10$ & -0.008267 & -0.22557 & -1.49645 \\
$N=20$ & -0.00828 & -0.22566 & -1.49677 \\
\hline
\end{tabular}

TABLE I

COMPARISON OF THE RESULTS FROM [5] AND THE ONES OBTAINED USING THE NUMERICAL INTEGRATION AND THE SERIES EXPANSION WITH $b=N R_{2}, N=10,20$ USING HYP_2F1.

\begin{tabular}{cccc}
\hline & Numerical & \multicolumn{2}{c}{ Series expansion } \\
& integration & $N=10$ & $N=20$ \\
\hline PFQ & - & 37.41 & 66.22 \\
HYP_2F1 & 1.36 & 0.06 & 0.11 \\
\hline
\end{tabular}

TABLE II

COMPARISON OF THE CPU TIMES (IN SECOND) NEEDED TO COMPUTE $Z$ FOR 100 FREQUENCIES BETWEEN $1 \mathrm{kHz}$ TO $100 \mathrm{kHz}$ FOR THE TWO FORMULATIONS (INTEGRAL EQUATION (11) AND SERIES EXPANSION (26)) USING DIFFERENT SUBROUTINES TO COMPUTE ${ }_{2} F_{1}$.

To illustrate the ability of the series expansion to provide good results at a lower computional cost than the numerical integration a comparison of the CPU times needed to compute the impedance for 100 frequencies between $1 \mathrm{kHz}$ to $100 \mathrm{kHz}$ every $1 \mathrm{kHz}$ is proposed. As exemplified in Table II the subroutine HYP_2F1 seems to be much more efficient than PFQ for our application. 


\section{B. A two-tanh profile case}

As already said ( $\S \mathrm{IV}$ ), in the two-tanh profile configuration we have not been able to find proper results in the literature to numerically validate our theoretical approach. A multi-layer model (described $\S$ IV) have then been developped and numerical results compared. Again with reference to Fig. 1 the parameters of the measurement configuration are: $r_{1}=2 \mathrm{~mm}, r_{2}=4 \mathrm{~mm}$, $h_{1}=0.5 \mathrm{~mm} h_{2}=7.3 \mathrm{~mm}$ and $N_{\text {turn }}=200$. According to (27) the conductivity profile has the following parameters: $\sigma_{1}=7 \times 10^{5} \mathrm{~S} \mathrm{~m}^{-1}, \sigma_{12}=5 \times 10^{5} \mathrm{~S} \mathrm{~m}^{-1}, \sigma_{2}=$ $8 \times 10^{5} \mathrm{~S} \mathrm{~m}^{-1}, c_{1}=0.2 \mathrm{~mm}, v_{1}=0.03 \mathrm{~mm}, c_{2}=$ $0.8 \mathrm{~mm}$ and $v_{2}=0.1 \mathrm{~mm}$. The variation of impedance $\Delta Z_{i}$ is computed at $N_{\text {freq }}$ frequencies equally distributed between $f_{\min }=100 \mathrm{kHz}$ and $f_{\max }=10 \mathrm{MHz}$ every $100 \mathrm{kHz}$.

Let us define the discrepency between a reference $\Delta \mathcal{Z}_{i}, i=1, \ldots, N_{\text {freq }}$ and an approximated value $\Delta Z_{i}$ taken as

$$
\operatorname{Err}=\frac{1}{N_{\text {freq }}} \sum_{i=1}^{N_{\text {freq }}} \frac{\left|\Delta Z_{i}-\Delta \mathcal{Z}_{i}\right|}{\left|\Delta \mathcal{Z}_{i}\right|}
$$

Comparison of variations of impedance obtained with the analytical model and those obtained with a numerical multi-layer model using the numerical integration is very good as reported in Fig. 3, corresponding CPU time and error being given in Table 3. Let us emphasize that best matching with the multi-layer model requires a number of, at least, $N=100000$ layers, which can be explained by the fact that, as described in $\S$ IV, the two multi-layer model parameters $d$ and $N$ are kept constant for all frequencies and whatever the variation of the conductivity profile.

The analytical formulation and numerical integration being validated let us now focus on the series expansion approach and its advantages. In the following only the analytical formulation using the subroutine HYP_2F1 to

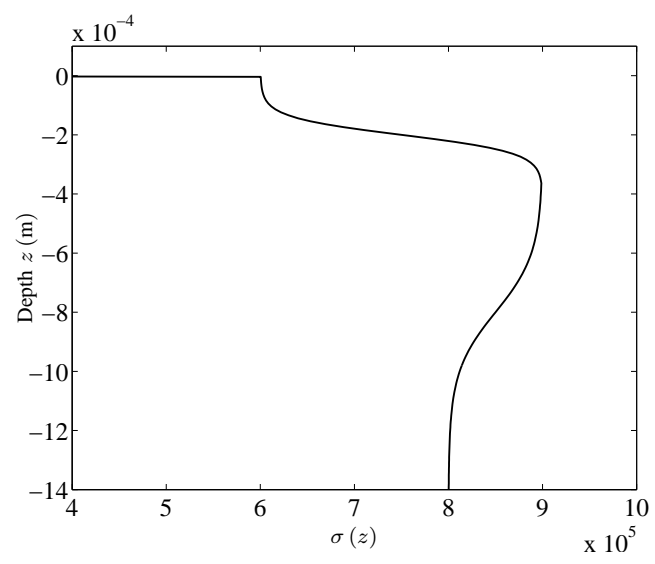

Fig. 2. Conductivity Profile.

\begin{tabular}{llrrr}
\hline & & & CPU Time (s) & Err (\%) \\
\hline HYP_2F1 & & & 6.63 & \multicolumn{1}{c}{-} \\
& $M=$ & 1000 & 24.48 & 795.0324 \\
Multi-layer & $M=$ & 10000 & 247.58 & 78.1864 \\
& $M=100000$ & 2506.05 & 7.8057 \\
& $M=1000000$ & 25087.74 & 0.7805 \\
\hline
\end{tabular}

TABLE III

COMPARISON OF THE CPU TIMES (IN SECOND) AND THE ERROR (IN \%) FOR THE COMPUTATION OF $Z$ USING A NUMERICAL INTEGRATION OF (11) WITH THE ANALYTICAL AND THE MULTI-LAYER MODELS.

compute ${ }_{2} F_{1}$ will be used and a comparison between the results using the numerical integration and the series expansion approach is provided in Fig. 4 and the corresponding CPU times and errors are reported in Table IV. It can be seen that $b$-which has been chosen as an integer multiple of $R_{2}$ as $b=N R_{2}$ - should be carefully chosen and a too small value, here $N=2$, leads to a large error whereas a higher value, $N=10$, gives very good results. As expected when $N$ increases the error between numerical integration and the series expansion decreases and tends to 0 which is paid for by an increase in computing time even if the "series" CPU time is still ten times lower (for $N=20$ ) than the "numerical 


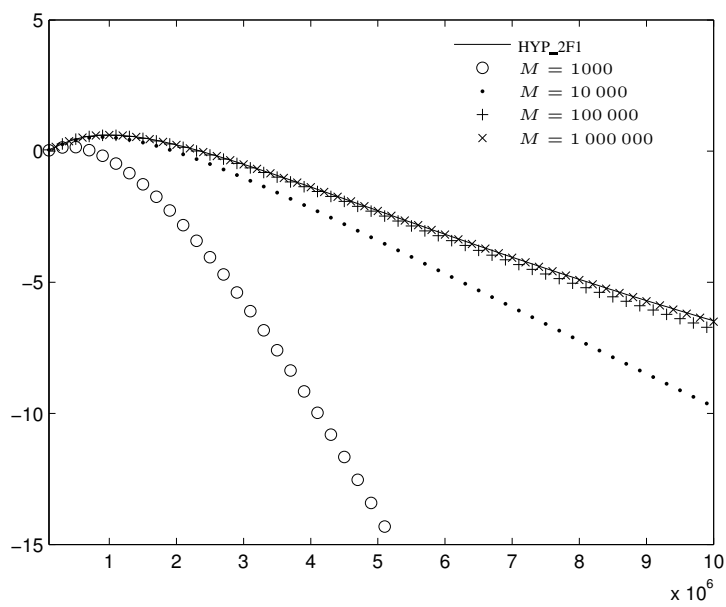

(a) Real Part as a function of the frequency (in $\mathrm{Hz}$ )

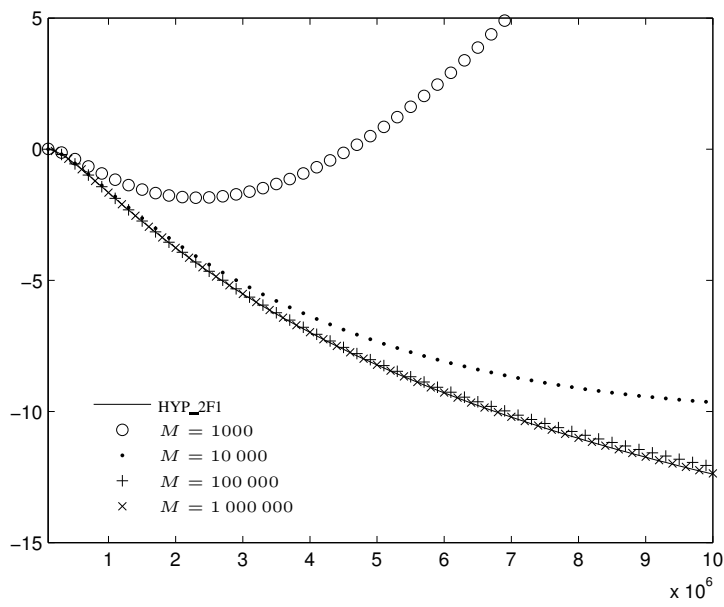

(b) Imaginary Part as a function of the frequency (in $\mathrm{Hz}$ )

Fig. 3. Comparison of results obtained with analytical and multi-layer models for different number of layers $N$.

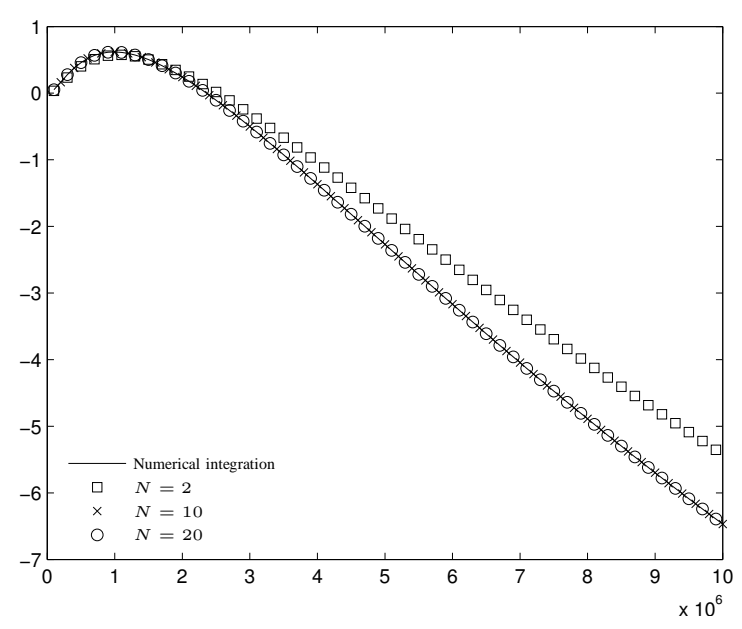

(a) Real Part as a function of the frequency (in $\mathrm{Hz}$ )

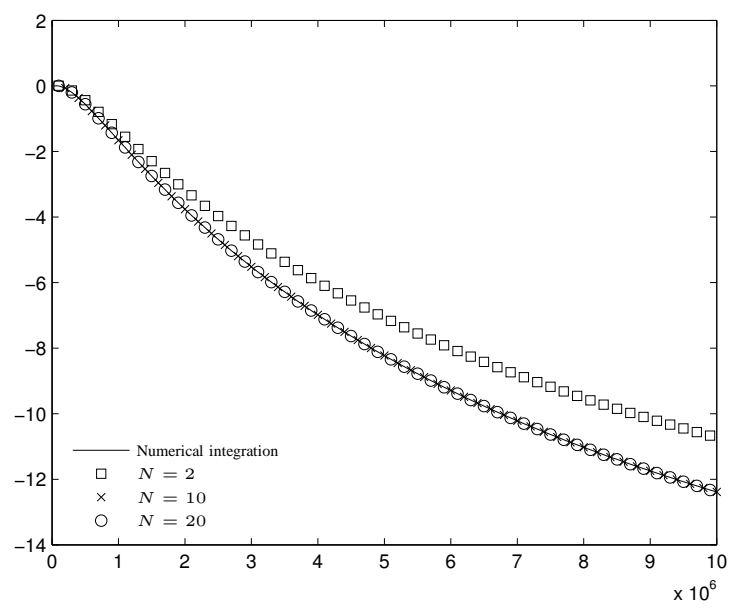

(b) Imaginary Part as a function of the frequency (in $\mathrm{Hz}$ )

Fig. 4. Comparison of the results obtained with analytical using a numerical integration and the series expansion with $b=N R_{2}, N=2,10,20$ with HYP_2F1.

integration" one.

\section{CONCLUSION}

In the context of development of decentralized power generation, it is of economical importance to improve the thermal efficiency of gas turbine by increasing the firing temperature. To optimize the maintenance cost of such advanced gas turbine, it is crucial to predict remaining coating life for reducing unexpected shut-downs caused by blade coating failure due to depletion of aluminum. Eddy Current Non-Destructive Evaluation can play a key role in assessing the conditions of turbine blade coating by estimating conductivity depth profiles. In this paper, we have reported on efforts towards that goal. We have developed the direct model of the impedance of an EC coil above a metal structure protected by a top overaluminized coating degraded by inward and outward diffusion of aluminum, assuming that the conductivity 


\begin{tabular}{lccc}
\hline & & CPU Time (in s) & Err (in \%) \\
\hline Numerical integration & & 6.63 & - \\
& $N=2$ & 0.11 & 15.3062 \\
\multirow{2}{*}{ Serie expansion } & $N=10$ & 0.40 & 0.0126 \\
& $N=20$ & 0.67 & 0.0011 \\
\hline
\end{tabular}

TABLE IV

COMPARISON OF THE CPU TIMES (IN SECOND) AND THE ERROR (IN \%) FOR THE TWO FORMULATIONS OF $Z$ (INTEGRAL EQUATION

(11) AND SERIES EXPANSION (26)) USING DIFFERENT VALUES $b=N R_{2}, N=2,10,20,{ }_{2} F_{1}$ BEING COMPUTED WITH HYP_2F1.

profile follows a two-hyperbolic-tangent law. The analysis of the approach as suggested is quite interesting, since the results obtained with this model are similar to those obtained with a numerical multi-layer model with a significant reduction of computing time.

\section{ACKNOWLEDGEMENTS}

The authors would like to thank Dominique Lesselier and Theodoros Theodoulidis and the two anonymous reviewers for their helpful comments.

\section{APPENDIX}

In the following and in the case of the two tanhprofil the analytical expressions of the general functions presented in (24) are given.

$$
\begin{aligned}
L= & \frac{\left[1-y_{2}(0)\right]^{\nu}}{y_{2}^{-\mu}(0)}{ }_{2} F_{1}\left(\mu+\nu, \mu+\nu+1 ; 2 \mu+1 ; y_{2}(0)\right) \\
M= & \frac{\left[1-y_{2}(0)\right]^{\nu}}{y_{2}^{\mu}(0)}{ }_{2} F_{1}\left(\nu-\mu+1, \nu-\mu ; 1-2 \mu ; y_{2}(0)\right) \\
N= & \frac{\left[y_{2}(0)-y_{2}^{2}(0)\right]}{v_{1}}\left[\frac{\left[\mu-(\mu+\nu) y_{2}(0)\right]}{y_{2}^{1-\mu}(0)\left[1-y_{2}(0)\right]^{1-\nu}}\right. \\
& \times{ }_{2} F_{1}\left(\mu+\nu, \mu+\nu+1 ; 2 \mu+1 ; y_{2}(0)\right) \\
& +y_{2}^{\mu}(0)\left[1-y_{2}(0)\right]^{\nu} \frac{(\mu+\nu)(\mu+\nu+1)}{2 \mu+1} \\
& \left.\times{ }_{2} F_{1}\left(\mu+\nu+1, \mu+\nu+2 ; 2 \mu+2 ; y_{2}(0)\right)\right]
\end{aligned}
$$

$$
\begin{aligned}
O= & \frac{\left[y_{2}(0)-y_{2}^{2}(0)\right]}{v_{1}}\left[\frac{(\nu+1-\mu)(\nu-\mu)}{y_{2}^{\mu}(0)(1-2 \mu)\left(1-y_{2}(0)\right)^{\nu}}\right. \\
& \times{ }_{2} F_{1}\left(\nu-\mu+2, \nu-\mu+1 ; 2-2 \mu ; y_{2}(0)\right) \\
& \frac{\left[1-y_{2}(0)\right]^{\nu-1}}{y_{2}^{\mu+1}(0)}\left[\mu-(\mu+\nu) y_{2}(0)\right] \\
& \left.\times{ }_{2} F_{1}\left(\nu-\mu+1, \nu-\mu ; 1-2 \mu ; y_{2}(0)\right)\right]
\end{aligned}
$$$$
P=\frac{\left[1-y_{2}(-r)\right]^{\nu}}{y_{2}^{-\mu}(-r)}{ }_{2} F_{1}\left(\mu+\nu, \mu+\nu+1 ; 2 \mu+1 ; y_{2}(-r)\right)
$$$$
Q=\frac{\left[1-y_{2}(-r)\right]^{\nu}}{y_{2}^{\mu}(-r)}{ }_{2} F_{1}\left(\nu-\mu+1, \nu-\mu ; 1-2 \mu ; y_{2}(-r)\right)
$$$$
R=\frac{\left[y_{2}(-r)-y_{2}^{2}(-r)\right]}{v_{1}}\left[\left[\mu-(\mu+\nu) y_{2}(-r)\right]\right.
$$$$
\times{ }_{2} F_{1}\left(\mu+\nu, \mu+\nu+1,2 \mu+1 ; y_{2}(-r)\right)
$$$$
+\frac{\left[1-y_{2}(-r)\right]^{\nu}}{y_{2}^{-\mu}(-r)} \frac{(\mu+\nu)(\mu+\nu+1)}{2 \mu+1}
$$$$
\left.\times{ }_{2} F_{1}\left(\mu+\nu+1, \mu+\nu+2 ; 2 \mu+2 ; y_{2}(-r)\right)\right]
$$$$
S=\frac{\left[y_{2}(-r)-y_{2}^{2}(-r)\right]}{v_{1}}\left[\frac{\left[1-y_{2}(-r)\right]^{\nu}}{y_{2}^{\mu}(-r)} \frac{(\nu+1-\mu)(\nu-\mu)}{-2 \mu+1}\right.
$$$$
\times{ }_{2} F_{1}\left(\nu-\mu+2, \nu-\mu+1 ; 2-2 \mu ; y_{2}(-r)\right)
$$$$
-\frac{\left[1-y_{2}(-r)\right]^{\nu-1}}{y_{2}^{\mu+1}(-r)}\left[\mu-(\mu-\nu) y_{2}(-r)\right]
$$$$
\left.\times{ }_{2} F_{1}\left(\nu-\mu+1, \nu-\mu ; 1-2 \mu ; y_{2}(-r)\right)\right]
$$

$$
\begin{aligned}
T= & \frac{(-r)\left[1-y_{3}(-r)\right]^{\tau}}{y_{3}^{-\lambda}} \\
& \times{ }_{2} F_{1}\left(\lambda+\tau, \lambda+\tau+1 ; 2 \lambda+1 ; y_{3}(-r)\right) \\
U= & \frac{\left[y_{3}(-r)-y_{3}^{2}(-r)\right]}{v_{2}}\left[\frac{\left[1-y_{3}(-r)\right]^{\tau-1}}{y_{3}^{1-\lambda}(-r)}\right. \\
& \times\left[\lambda-(\lambda+\tau) y_{3}(-r)\right] \\
& \times{ }_{2} F_{1}\left(\lambda+\tau, \lambda+\tau+1 ; 2 \lambda+1 ; y_{3}(-r)\right) \\
& +\frac{\left[1-y_{3}(-r)\right]^{\tau}}{y_{3}^{-\lambda}(-r)} \frac{(\lambda+\tau)(\lambda+\tau+1)}{2 \lambda+1} \\
& \left.\times{ }_{2} F_{1}\left(\lambda+\tau+1, \lambda+\tau+2 ; 2 \lambda+2 ; y_{3}(-r)\right)\right]
\end{aligned}
$$

$\mu, \nu, y_{2}, y_{3}$ are defined in (29). 


\section{REFERENCES}

[1] T. Theodoulidis and J. Bowler, "Interaction of an eddy-current coil with a right-angled conductive wedge," IEEE Trans. Magn., vol. 46, no. 4, pp. $1034-1042$, apr. 2010.

[2] A. Skarlatos and T. Theodoulidis, "Impedance calculation of a bobbin coil in a conductive tube with eccentric walls," IEEE Trans. Magn., vol. 46, no. 11, pp. 3885 -3892, nov. 2010.

[3] K. Ishida, T. Itaya, A. Tanaka, and N. Takehira, "Magnetic field analysis of an arbitrary shaped coil using shape functions," IEEE Trans. Magn., vol. 45, no. 1, pp. 104 -112, jan. 2009.

[4] D. Mayer, "Inductance of coil in nonhomogenous surrounding," IEEE Trans. Magn., vol. 45, no. 3, pp. 1032 -1035, mar. 2009.

[5] E. Uzal and J. Rose, "The impedance of eddy current probes above layered metals whose conductivity and permeability vary continuously," IEEE Trans. Magn., vol. 29, no. 2, pp. 1869-1873, March 1993.

[6] E. Uzal, J. Moulder, S. Mitra, and J. Rose, "Impedance of coils over layered metals with continuously variable conductivity and permeability : theory and experiment," J. Appl. Phys., vol. 74, no. 3, pp. 2076-2089, August 1993.

[7] E. Uzal, J. Moulder, and J. Rose, "Experimental determination of the near-surface conductivity profiles of metal from electromagnetic induction (eddy current) measurements," Inverse Prob., vol. 10, pp. 753-764, 1994.

[8] T. Theodoulidis, T. Tsiboukis, and E. Kriezis, "Analytical solutions in eddy current testing of layered metals with continuous conductivity profiles," IEEE Trans. Magn., vol. 31, no. 3, pp. 2254-2260, 1995.

[9] C. Dodd and W. Deeds, "Analytical solutions to eddy-current probe-coil problems," J. Appl. Phys., vol. 39, no. 6, pp. 28292838, 1968.

[10] C. Cheng, C. Dodd, and W. Deeds, "General analysis of probe coils near stratified conductors," Int. J. Nondestruct. Test., vol. 3, pp. 109-130, 1971.

[11] K. Schmidt, O. Sterz, and R. Hiptmair, "Estimating the eddycurrent modeling error," IEEE Trans. Magn., vol. 44, no. 6, pp. $686-689$, june 2008.

[12] A. A. Kolyshkin, A. P. Smolyakov, and R. Vaillancourt, "Analytical solution in eddy-current testing of double-layer media with depth-varying magnetic properties," IEEE Trans. Magn., vol. 27, no. 5, pp. 4360-4365, 1991.

[13] A. Kolyshkin and R. Vaillancourt, "Analytical solutions to eddycurrent testing problems for a layered medium with varying properties," IEEE Trans. Magn., vol. 33, no. 4, pp. 2473 -2477, jul 1997.

[14] T. Theodoulidis and E. Kriezis, "Series expansions in eddy current nondestructive evaluation models," J. Mater. Process. Technol., vol. 161, no. 1-2, pp. 343-347, Apr. 2005.

[15] M. Abramowitz and I. A. Stegun, Handbook of Mathematical Functions. Washington, D.C.: National Bureau of Standards, 1966.

[16] W. F. Perger, A. Bhalla, and M. Nardin, "A numerical evaluator for the generalized hypergeometric series," Comput. Phys. Commun, vol. 77, no. 2, pp. 249-254, Oct. 1993. [Online]. Available: http://www.sciencedirect.com/science/article/B6TJ546G8B8K-4C/2/8e736a6df08afef03b3de87b37d13f92

[17] N. Michel and M. Stoitsov, "Fast computation of the Gauss hypergeometric function with all its parameters complex with application to the Pöschl-Teller-Ginocchio potential wave functions," Comput. Phys. Commun, vol. 178, no. 7, pp. 535-551, Apr. 2008. [Online]. Available: http://www.sciencedirect.com/science/article/B6TJ54R8H1W9-3/2/46d74d91aa6802f2434e93b0f6ffcf87 